\section{Wacker verstärkt Präsenz in Mittel- und Südamerika}

Die Wacker Chemie AG investiert rund 1,2 Mio. € für den Ausbau ihres bestehenden Technical Centers in São Paulo (Brasilien) und die Gründung eines neuen Technical Centers in Mexiko Stadt (Mexiko). Auch das internationale Schulungszentrum des Konzerns - die Wacker Academy - wird an beiden Standorten erweitert bzw. neu eröffnet.

D as Unternehmen möchte dadurch den Bedürfnissen seiner Kunden in den stark wachsenden Märkten der Region Rechnung tragen und seine lokale Kompetenz in Forschung, Entwicklung und Anwendungstechnik steigern. Beide Projekte sollen im 1. Quartal 2013 fertiggestellt werden.

„Mittel- und Südamerika sind wichtige Zukunftsmärkte für Wacker. Unser Umsatz ist in diesen Regionen in den vergangenen fünf Jahren um durchschnittlich 15 Prozent pro Jahr gewachsen“, so Vorstandsvorsitzender Dr. Rudolf Staudigl. „Die zunehmende Nachfrage nach technologisch hochwertigen Lösungen lässt für unser Silicon- und Polymergeschäft in den kommenden Jahren weiterhin Steigerungsraten erwarten. Den wachsenden Bedarf unse- rer Kunden begleiten wir mit dem Ausbau unseres Serviceangebots vor Ort“, begründet Staudigl den Ausbau der Aktivitäten in Mittel- und Südamerika.

Schwerpunkt der Erweiterung des Technical Center in Jandira nahe São Paulo sind die Labore für polymere Dispersionspulver, die zum Beispiel in Trockenmörteln für Betonanwendungen, Fliesenklebstoffen oder Dichtungsschlämmen zum Einsatz kommen. Auch in Mexiko stehen polymere Bindemittel für Farben, Beschichtungen oder Klebstoffe im Mittelpunkt des neuen Technical Centers. Von hier aus sollen künftig Kunden aus Mexiko, Belize, Guatemala, El Salvador, Honduras, Nicaragua, Costa Rica, Panama, der Karibik, Kolumbien, Ecuador, Peru, Venezuela und Bolivien betreut werden. Das Technical Center in Brasilien dient weiterhin als südamerikanische

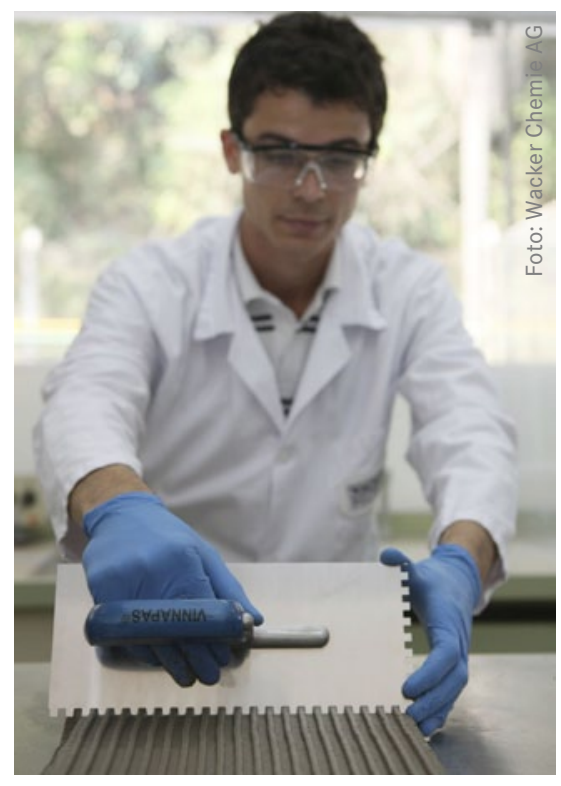

Im Technical Center Wacker Química do Brasil testen Anwendungstechniker Produkte und Formulierungen für den südamerikanischen Markt.

Schwerpunkt des Ausbaus sind die Labore für polymere Dispersionspulver, die zum Beispiel in Trockenmörteln für Betonanwendungen, Fliesenklebstoffe oder Dichtungsschlämmen zum Einsatz kommen.

Drehscheibe für Kunden aus Argentinien, Brasilien, Chile, Paraguay und Uruguay.

\title{
29. TKB-Fachtagung „Klebstoffe in der Fußbodentechnik“
}

Am 19. März 2013 findet zum 29. Mal in Frankfurt die von der Technischen Kommission Bauklebstoffe (TKB im IVK, Düsseldorf) veranstaltete Tagung „Klebstoffe in der Fußbodentechnik“ statt.

$\mathrm{D}$ iese jährliche Veranstaltung dient der Weitergabe aktueller Informationen und dem Meinungsaustausch über Themen und Fragestellungen aus den Bereichen

- Klebstoffe und Klebverfahren im Bereich der Fußbodentechnik,

- Eigenschaften und Wechselwirkungen von Belägen,

- Untergründen und Verlegewerkstoffen,

- Arbeits- und Umweltschutz bei Bodenbelag- und Parkettarbeiten sowie

- Ursachen und Vermeidung von Reklamationen.

Geboten werden den Teilnehmern diesmal insgesamt acht Fachvorträge u.a. über die mechanischen Eigenschaften von Parkettklebstoffen bei Feuchtigkeitsbelastung, über wässrige Kontaktklebung für anspruchsvolle Anwendungen und das Parkettkleben auf Fertigteilestrichen.

Außerdem wird der Frage nachgegangen, wie nachhaltig Verlegewerkstoffe sein können und auf welche Weise sich Gebäude bewerten und zertifizieren lassen.

Darüber hinaus erhalten die Teilnehmer Informationen über den aktuellen Stand der Technik bei Calciumsulfatestrichen.
Ein Beitrag über Erfahrungen, die bei der Fußbodengestaltung von Demenz-Wohnbereichen gesammelt wurden, und Ergebnisse einer Branchenumfrage runden die Veranstaltung ab, die sich in erster Linie an alle Fachleute auf dem Gebiet der Bodenbelagsarbeiten, Klebstoff- und Rohstoffhersteller sowie Sachverständige, Institute, Planer und Architekten wendet.

Weitere Infos: Industrieverband Klebstoffe e.V., D-40219 Düsseldorf, Tel.: +49 / (0)211 / 67931-14, martina.weinberg@klebstoffe.com, www.klebstoffe. com. 